\title{
Dark Line Detection with Line Width Extraction
}

\author{
Qin Li, Lei Zhang, Jane You, and David Zhang \\ Department of Computing \\ The Hong Kong Polytechnic University \\ KLN, Hong Kong \\ \{csqinli,cslzhang\}@comp.polyu.edu.hk
}

\begin{abstract}
Automated line detection is a classical image processing topic with many applications such as road detection in remote images and vessel detection in medical images. Many traditional line detectors, such as Gabor filter, the second order derivative of Gaussian and Radon transform will response not only to lines but also to edges, e.g. they will give high responses to the edges of bright lines or blobs when only dark lines are required. To reduce false detections when extracting only dark (or bright) lines, in this paper we propose a line detector by using the first derivative of Gaussian. It can detect dark lines without much false detection on blobs or bright lines. Meanwhile, the proposed method can estimate line width simultaneously. Experiments on various images are performed to test the proposed algorithm.
\end{abstract}

Index Terms - Line detection, width estimation, edge detection

\section{Introduction}

Automated line detection in digital images is a classical problem with many applications such as road detection in remote sensing images and vessel detection in medical images. Many line detection algorithms has been proposed such as local contrast analysis based methods [1-3], parallel edges seeking based methods [4-5], and ridge seeking based methods [6-12]. However, these line detectors response not only to lines but also to edges. For instance, they will give high responses to the edges of bright lines or blobs when we only want to detect dark lines. In addition, most of the existing line detectors are not good at estimating line width.

In [9-10], the real part of Gabor filter was used to enhance ridges in an image. However, similar to other band-pass filters such as the second order derivative of Gaussian [8], it responses to not only lines but also edges. This will cause false detections in many images. Another problem of these filters is the scale selection of the filter. In order to detect lines of arbitrary widths, it is often necessary to iterate the detection procedure in the scale space. Conventionally, the lines in an image are detected using a single optimal scale or using the maximum

\author{
Prabir Bhattacharya \\ Institute for Information Systems Engineering \\ Concordia University \\ Quebec, Canada \\ prabir@ciise.concordia.ca
}

response of all scales. Nonetheless this will lead to overestimation of the line width of small lines. The Hough transform [11] and Radon transform [12] based methods are not good at either enhancing lines or estimating line width. They may need preprocessing to help line detection and those preprocessing may cause false detections.

In $[8,12]$, methods to estimate line width were proposed by analyzing the filter responses. These methods can obtain precise line width. However, they still have the problem of false detections on edges. In [5], a pair of line detectors was used to find the parallel edges. By combing the responses of a pair of line detectors, it will eliminate some false detection. However this technique is not effective enough to eliminate false detection due to the limitation of scales (small bright lines will be false detected by large scale filters when dark lines are required) and it may under-estimates line widths of large lines.

This paper presents a line detection method based the first order derivative of Gaussian. Without loss of generality, we focus on dark line detection. The detection of bright lines can be done by reverse the image gray level. The proposed method can not only detect dark lines without much false detection on blobs or bright lines but also estimate line width simultaneously.

The reset of the paper is organized as follows. Section 2 presents the dark line detection algorithm detailedly. Section 3 presents experimental results and Section 4 concludes the paper.

\section{The Line Detection Algorithm}

Traditional line detectors response not only to lines but also to edges [1-11]. For example, the second order derivative of Gaussian (SODG) [8] is defined as

$$
g_{\sigma}^{\prime \prime}(x)=\frac{x^{2}-\sigma^{2}}{\sqrt{2 \pi} \sigma^{5}} e^{-\frac{x^{2}}{2 \sigma^{2}}}
$$

Fig. 1 shows the output by convolving SODG with a 1-D signal. Fig. 1(a) shows a 1-D signal with the cross section of a dark line, a bright line and a large dark blobs. Fig. 1(b) is the profile of SODG filter. Fig. 1(d) shows the filter response by convolving the SODG with the 1-D signal. We can see that SODG will enhance the dark line but it will also produce high responses to the edges of bright lines and blobs. Therefore, false detections will arise. 
In [13], a first order derivative of Gaussian (DOG) based method was used to detect the center of a line. Inspired by that idea, we propose here a novel line detection method which can eliminate the false detections caused by edges and estimate line width precisely.

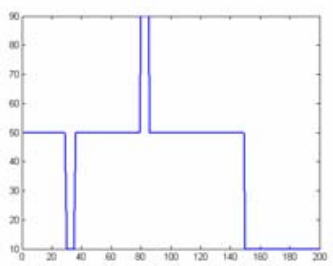

(a)

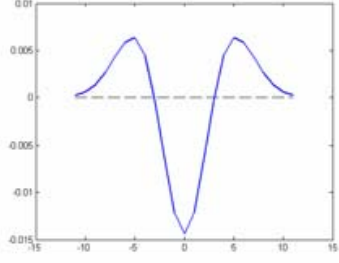

(b)

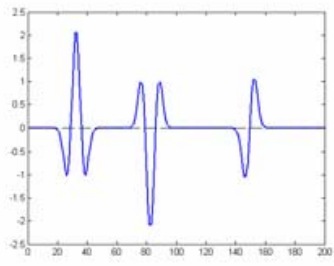

(d)

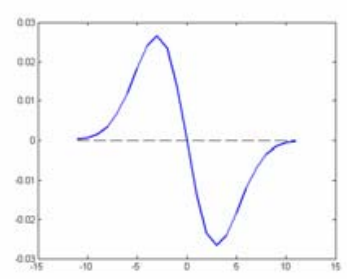

(c)

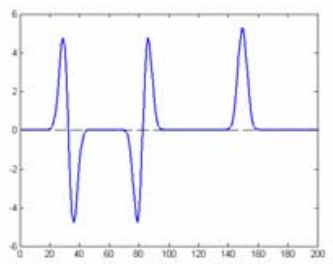

(e)
Figure 1. (a) 1-D signal with the cross section of a dark line, a bright line and a large dark blob; (b) \& (c) are the second and first order derivatives of Gaussian; (d) \& (e) are the filter responses to (a) by (b) and (c).

The DOG filter is defined as

$$
g_{\sigma}^{\prime}(x)=\frac{-x}{\sqrt{2 \pi} \sigma^{3}} e^{-\frac{x^{2}}{2 \sigma^{2}}}
$$

Fig. 1(c) shows the shape of DOG and Fig. 1(e) shows its response to the 1-D signal in Fig. 1(a). By observing the filter response, we found there are two advantages of line detection using DOG. First, the zero-crossings of dark line and bright line go oppositely, i.e. one is positive-tonegative and the other is negative-to-positive. This information can be used to separate bright line and dark line. Second, the distance between the local maximum and local minimum can be used to estimate the line width. If a proper filter scale is used, the line width can be estimated precisely. With those observations, we propose a new DOG based line detection method.

We define a 2-D DOG filter as

$$
g_{i}(x, y)=\frac{-x}{\sqrt{2 \pi} \sigma_{i}^{3}} e^{-\frac{x^{2}}{2 \sigma_{i}^{2}}}, \text { for }|x| \leq 3 \sigma_{i}, \quad|y| \leq L_{i} / 2
$$

where $\sigma_{i}$ is the standard deviation of the Gaussian function at scale $i$ and $L_{i}$ is the length of the filter in $y$ direction at that scale. Parameter $L_{i}$ is used to smooth a line along its tangent direction.

The rotation of $g_{i}(x, y)$ with angle $\phi$ is then implemented by using $g_{i}^{\phi}\left(x^{\prime}, y^{\prime}\right)=g_{i}(x, y)$, where $x^{\prime}=x \cos \phi+y \sin \phi$ and $y^{\prime}=y \cos \phi-x \sin \phi$.
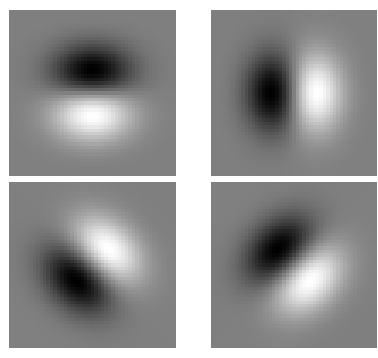

Figure 2. 2-D directional first order derivative of Gaussian filters.

Fig. 2 shows the 2-D DOG filter at four directions. Without loss of generality and for the convenience of expression, we only discuss the filter in the horizontal direction. The filters in other directions can be derived similarly. The response of a filter $g_{i}(x, y)$ to an input image $f(x, y)$ can be expressed by

$$
R_{i}(x, y)=g_{i}(x, y) * f(x, y)
$$

The centerline response can be defined by

$$
\begin{aligned}
& C_{i}(x, y)= \\
& \left\{\begin{array}{cc}
R_{i}(x-d, y)+\left|R_{i}(x+d, y)\right|, & \text { If } R_{i}(x-d, y)>0 \& R_{i}(x+d, y)<0 \\
0, & \text { Otherwise }
\end{array}\right.
\end{aligned}
$$

where $d$ is used to obtain the magnitude around the zerocrossing. The centerline response is then double thresholded [13] to obtain the centerline. The line direction is determined as the direction with maximal centerline response. Then the line width can be estimated by finding the distance between local maximum and local minimum along the perpendicular direction of the line.

Once we obtain the center of a line, the direction of a line and the width of a line, the line can be interpolated through this information. Fig. 3 illustrates the line detection by the proposed method in comparison with the SDOG method. To produce high responses at the center of both small lines and large lines, we implement the 2-D DOG filtering at several scales and use the maximal response of all scales. Figs. 3(b) and (c) show the filter responses of the SDOG and the proposed DOG, respectively. We can see that the SDOG gives high responses to both bright lines and dark lines and the proposed DOG responses only to dark lines. By thresholding the filter response of the SDOG, the obtained 
segmentation result is shown in Fig. 3(d). Fig. 3(e) shows the segmentation result of the proposed method, which is obtained by interpolating lines by their centers, widths, and directions. We can see that the proposed method can detect dark lines without false detections on blobs or bright lines and it can achieve better line width estimation simultaneously.

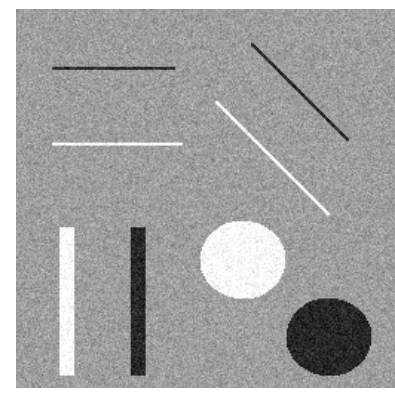

(a)

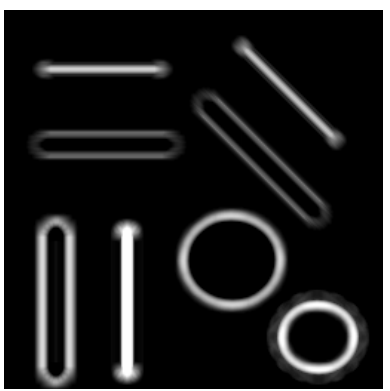

(b)

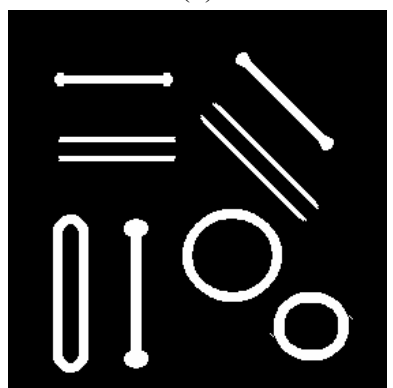

(d)

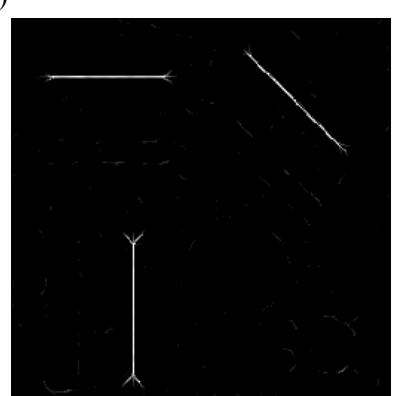

(c)

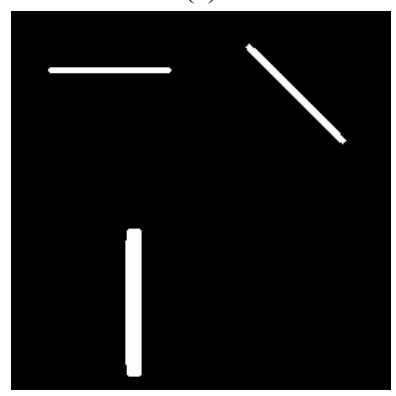

(e)
Figure 3. (a) 2-D image with dark lines, bright lines and large blobs; (b) \& (c) are the filtering responses by the second and first order derivatives of Gaussian; (d) \& (e) are the segmentation results by the second order derivative of Gaussian and the proposed method.

\section{Experimental Results}

We tested our method on a medical image and a remote sensing image. Fig. 4 shows the line detection using the proposed method on a medical image in Fig. 4(a), which contains dark lines and bright blobs. Fig. 4(b) shows the filtering output of the SDOG by keeping the maximal response of all scales. There are many high responses to the edges of bright blobs, which are hard to remove by thresholding. Fig. 4(c) shows the filter response by the proposed DOG filter. Figs. 4(d) and (e) show the segmentation results by the two schemes. Only dark lines, i.e. the desired vessels, are segmented by the proposed method. The line widths of small lines estimated by the proposed method are also more accurate than the SDOG method, which tends to over-estimate the widths of small lines.

Fig. 5 shows another example of line detection in a remote sensing image shown in Fig. 5(a). This image contains bright lines and dark blobs. By calculating the complemental image, the proposed method can then be applied. It can be seen that the proposed method obtained much better result than the SDOG method in both the detection of desired lines and the accuracy of line width.

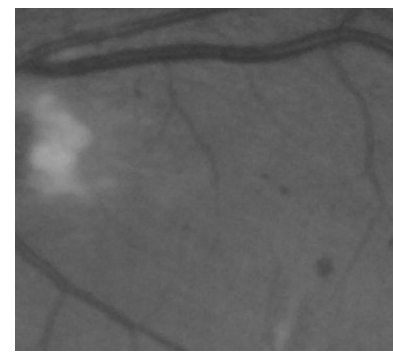

(a)

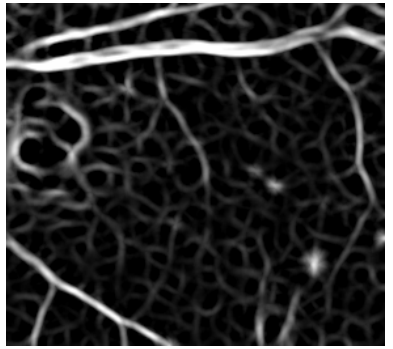

(b)

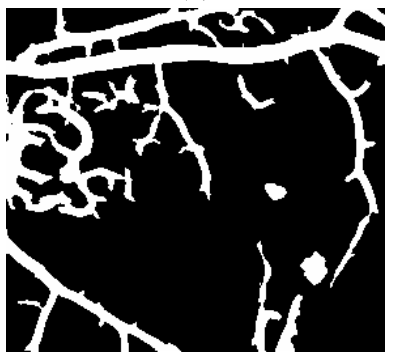

(d)

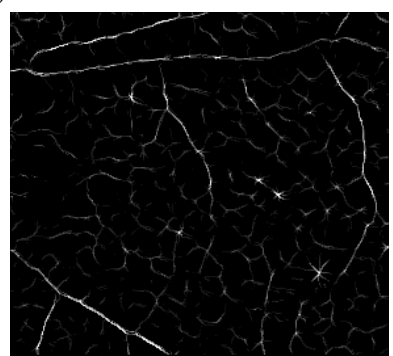

(c)

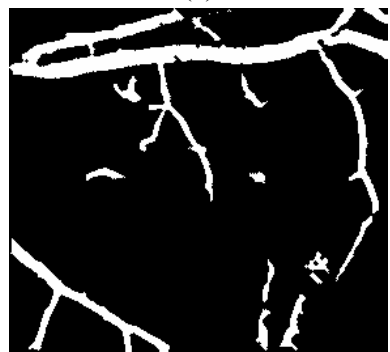

(e)
Figure 4. (a) A retinal image with dark lines and bright blobs; (b) \& (c) are the filtering responses by the second and first order derivatives of Gaussian; (d) \& (e) are the segmentation results by the second order derivative of Gaussian and the proposed method.

\section{Conclusion and Further Work}

We proposed a line detection method by using the first order derivative of Gaussian. By finding the center of a line, the direction of a line and the width of a line, the proposed method can detect dark lines without much false detection on the edges of blobs or bright lines. Meanwhile, the proposed scheme can estimate line width precisely. 
However, the proposed method is relatively sensitive to noise so that its robustness is to be improved. The interpolating procedure also costs much time and a fast algorithm needs to be developed to interpolate lines smoothly and quickly. Those will be the focuses in our future research work.

\section{References}

[1] M.A. Fischler, "The Perception of Linear Structure: A Generic Linker," Image Understanding Workshop, pp. 1,565-1,579. San Francisco: Morgan Kaufmann Publishers, 1994.

[2] D. Geman and B. Jedynak, "An Active Testing Model for Tracking Roads in Satellite Images," IEEE Trans. Pattern Analysis and Machine Intelligence, vol. 18, no. 1, pp. 1-14, Jan. 1996.

[3] Mark J. Carlotto, "Enhancement of Low-Contrast Curvilinear Features in Imagery", IEEE Transactions On Image Processing, Vol. 16, No. 1, pp. 221-228, January 2007.

[4] J.B. Subirana-Vilanova and K.K. Sung, "Multi-Scale Vector-Ridge- Detection for Perceptual Organization Without Edges," A.I. Memo 1318, MIT Artificial Intelligence Lab., Cambridge, Mass., Dec. 1992.

[5] T.M. Koller, G. Gerig, G. Székely, and D. Dettwiler, "Multiscale Detection of Curvilinear Structures in 2-D and 3-D Image Data," Fifth Int'l Conf. Computer Vision, pp. 864-869. Los Alamitos, Calif: IEEE CS Press, 1995.

[6] L.A. Iverson and S.W. Zucker, "Logical/Linear Operators for Image Curves," IEEE Trans. Pattern Analysis and Machine Intelligence, vol. 17, no. 10, pp. 982-996, Oct. 1995.

[7] J.B.A. Maintz, P.A. van den Elsen, and M.A. Viergever, "Evaluation of Ridge Seeking Operators for Multimodality Medical Image Matching," IEEE Trans. Pattern Analysis and Machine Intelligence, vol. 18, no. 4, pp. 353-365, Apr. 1996.

[8] C. Steger, "An unbiased detector of curvilinear structures," IEEE Trans. Pattern Anal. Machine Intell., vol. 20, pp. 113-125, Feb. 1998.

[9] J. H. Van Deemter, J. M. H. Du Buf, "Simultaneous Detection Of Lines And Edges Using Compound Gabor Filters", International Journal of Pattern Recognition and Artificial Intelligence, Vol. 14, No. 6, pp. 757-777, 2000.

[10] Z.-Q. Liu, J. Cai, and R. Buse, Handwriting Recognition: Soft Computing and Probabilistic Approaches, pp. 31-57, Springer, Berlin, 2003.

[11] R. C. Gonzales and R. E. Woods, Digital Image Processing, Reading: Addison-Wesley Publishing Co., 1993.

[12] Qiaoping Zhang and Isabelle Couloigner, "Accurate Centerline Detection and Line Width Estimation of Thick Lines Using the Radon Transform", IEEE Transactions On Image Processing, Vol. 16, No. 2, pp. 310-316, February 2007.

[13] A. M. Mendonça, and A. Campilho, "Segmentation of Retinal Blood Vessels by Combining the Detection of Centerlines and Morphological Reconstruction," IEEE Trans. Med. Imag., vol. 25, no. 9, pp. 1200-1213, Sep. 2006.
[14] J. Canny, "A computational approach to edge detection," IEEE Transactions on Pattern Recognition and Machine Intelligence, vol. PAMI-8, pp. 679-698, Nov. 1986.

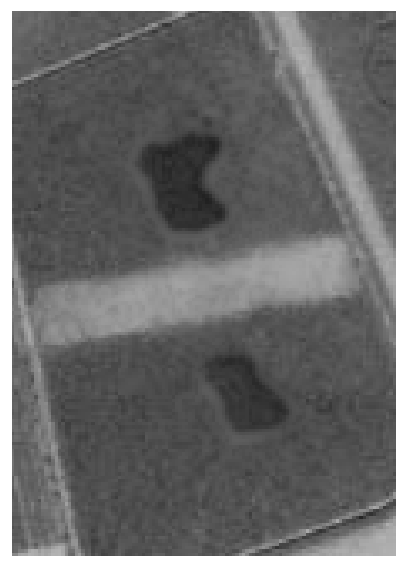

(a)

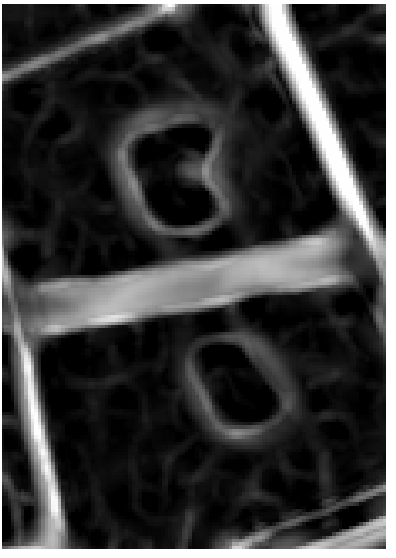

(b)

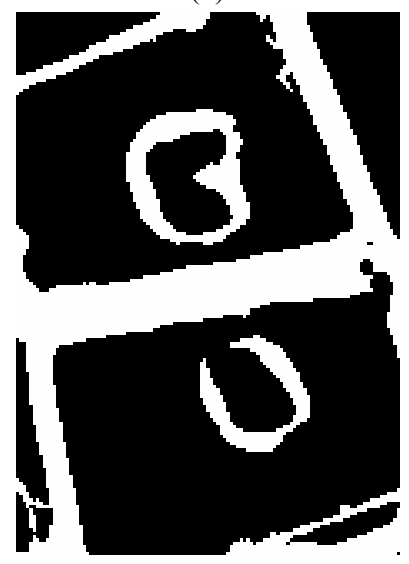

(d)

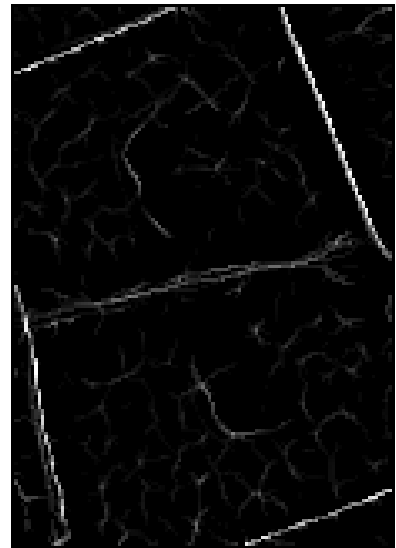

(c)

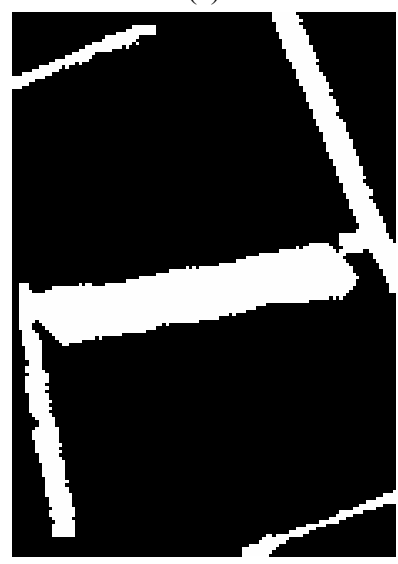

(e)
Figure 5. (a) A remote sensing image with bright lines and dark blobs; (b) \& (c) are the filtering responses by the second and first order derivatives of Gaussian; (d) \& (e) are the segmentation results by the second order derivative of Gaussian and the proposed method. 